\title{
A Comparative Study of the Iterative Numerical Methods Used in Mine Ventilation Networks
}

\author{
B. Maleki* \\ Department of Mining Engineering \\ Imam Khomeini International University \\ Qazvin, Iran
}

\author{
E. Mozaffari \\ Department of Mining Engineering \\ Imam Khomeini International University \\ Qazvin, Iran
}

\begin{abstract}
Ventilation is one of the key safety tasks in underground mines. Determination of the airflow through mine openings and ducts is complex and often requires the application of numerical analysis. The governing equations used in the computation of mine ventilation are discussed in matrix forms. The aim of this paper is to compare the most frequently used numerical analysis methods, which includes Newton-Raphson and the Linear Theory. It is the challenge of this study to investigate the influence of the initial flow rates and the fans in the network. A simulated mine ventilation network is represented in order to examine the two numerical methods. The numerical results acquired from Newton-Raphson method exhibited faster rate of convergence in comparison to those of the Linear Theory method. The mine ventilation networks are less expanded, therefore, the Newton-Raphson method converges faster. On the other hand, when using computational tools and software the advantage of faster convergence becomes less important, and therefore the Linear Theory method will be more preferred.
\end{abstract}

Keywords-mine ventilation; network analysis; NewtonRaphson method; linear theory

\section{INTRODUCTION}

Mine networks often comprise a number of loops and nodes connecting all branches. The airflow and air pressure distribution in mine openings and ducts can be worked out through solving the governing equations based on conservation Theory. The objective of the mine ventilation network analysis is twofold: a) the airflows and pressure distribution around the network are determined; b) the energy losses associated with all branches including those with fans and regulating doors are calculated. The laws of mass and energy conservation are used for the achievement of these objectives. The earliest numerical method, which is primarily used in water distribution network design, is that of Hardy-Cross method [1]. This method has also been used for mine ventilation network analysis [2]. The method was further developed to Newton-Raphson method to speed up the rate of convergence. In order to satisfy continuity, some initial values may be given to the airflows. The accuracy of these estimates will affect the speed of convergence. A new method was proposed by civil engineering practitioners in designing hydraulic distribution networks [3]. It is called the Linear Theory method and also relies on approximate evaluation techniques, which include functional approximation. The method was further extended by adding pumps and reservoir to the network [4], and later applied to mine ventilation networks by Bhamidipati and Procarione [5].

The governing equations may be combined and shown in the forms of matrixes. Kolarczyk [6] discussed on the application of matrix analysis methods in determining the changes due to the resistances and air flows of branches. The operations research methods were used in analysis and design of mine ventilation systems [7]. A simulation and optimization software for mine ventilation system of underground mines was programmed [8]. The use of 3D simulation system in mine ventilation management was also reported [9]. In this paper, first the key equations used for the ventilation networks analysis in the matrix forms will be represented. Then, the two approximate evaluation techniques, the Newton-Raphson and the Linear Theory methods, will be compared. In order to disclose some of the most significant features of the two methods, an example of a simulated mine ventilation network problem will be solved to demonstrate the applicability of the iterative numerical methods used. It will aim at demonstrating the difference between the approximation methods, the associated convergence properties in particular. The matrix form problem solving approach will be useful for the development of mine ventilation network algorithms [8,9]. The nature of oscillations in successive estimates and the way each method reaches the converged solution will be examined and discussed. Attempts will be made to show which method works more efficiently for mine ventilation networks.

\section{Mine Ventilation NeTWORKS}

Mine ventilation networks generally consist of a number of loops, where each loop has a number of branches. A simple form of a mine ventilation network is shown in Figure 1. 


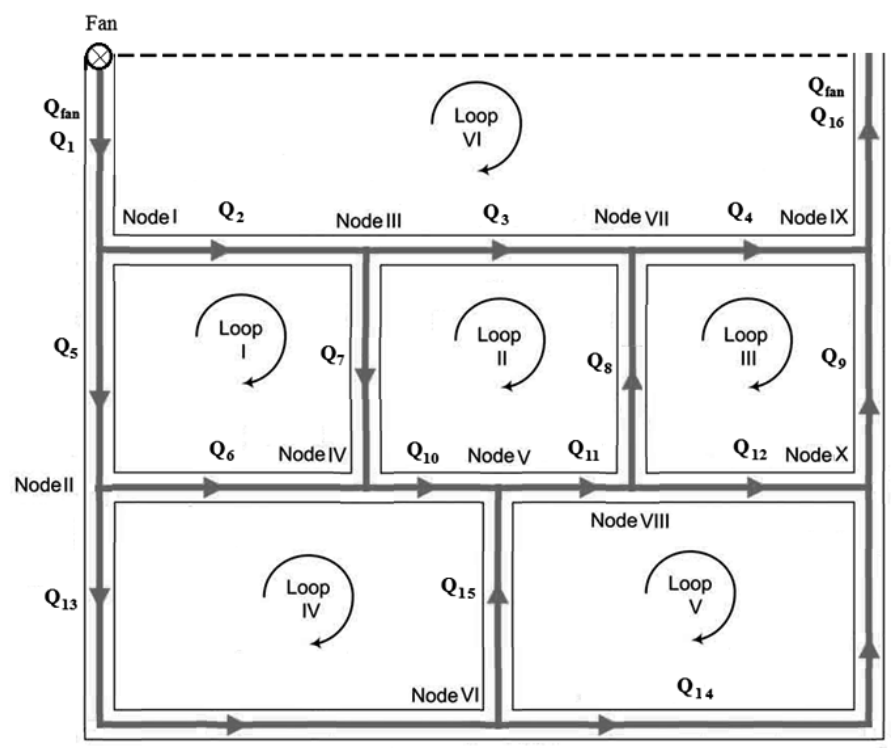

Fig. 1. An example of a simulated mine ventilation network

The branches join at nodes. Each branch represents one of the mine openings that may intersect with other openings at two ends. The network includes 5 actual loops as well as a virtual loop. It also includes 16 branches and 10 nodes. There will be lack of information about the air flows in all branches and the pressure drops concerned. Thus, the aim is to initially work out the airflows using the governing equations and taking into account the characteristics of the network. The matrix form approach will provide useful data, which then could be used in mine ventilation network algorithm and system developments [9].

Equations 1 and 2 are the governing equations used for the analysis of the ventilation networks. The first equation is satisfied for each branch to introduce the associated pressure drop, while the second equation is satisfied for each node according to the continuity law. In eq. $2, j$ represents the total number of nodes while $(j-1)$ is the number of independent nodes. Other parameters are $n$ : the number of branches, $R_{i}$ : the resistance, $Q_{i}$ : the airflow and $\Delta P_{i}$ : the pressure drop due to the airflow in branches [10]

$$
\begin{aligned}
& \Delta P_{i}=R_{i} Q_{i}^{2}(i=1,2, \ldots, n) \\
& \sum_{i=1}^{n} a_{k i} Q_{i}=0(k=1,2, \ldots, j-1)
\end{aligned}
$$

A node matrix may be represented, as shown in equation 3 , in which $a_{k i}$ is the general element of the matrix [11]. It equals 1 if the flow direction is such that it passes through branch $i$ and enters node $k$. But, it equals -1 if the air goes through branch $i$ after it leaves node $k$. When there is no intersection between the branch and node $a_{k i}$ is considered to be zero.

$$
A=\left[\begin{array}{cccccc}
a_{11} & a_{12} & \ldots & a_{1 i} & \ldots & a_{1 n} \\
\ldots & \ldots & \ldots & \ldots & \ldots & \ldots \\
a_{k 1} & \ldots & \ldots & a_{k i} & \ldots & a_{k n} \\
\ldots & \ldots & \ldots & \ldots & \ldots & \ldots \\
a_{(j-1) 1} & \ldots & \ldots & a_{(j-1) i} & \ldots & a_{(j-1) n}
\end{array}\right]
$$

Equations (1) and (2) are used if the unknowns are the branches' airflows, but if the pressures at nodes are the unknowns these equations should be rearranged for pressure [12].

Equation (4) can be applied to each loop:

$$
\sum_{i=1}^{n} C_{o i} \Delta P_{i}=0(o=1,2, \ldots, l)
$$

where $l$ is the number of loops, and the values of $C_{o i}$ (the elements of the matrix in equation 5) are defined as: $C_{o i}=1$ if branch $i$ is contained in loop $o$, and possesses the same flow direction, $C_{o i}=-1$ if branch $i$ is contained in loop $o$, but has the opposite flow direction, and $C_{o i}=0$ if branch $i$ is not contained in loop $o$.

$$
B=\left[\begin{array}{cccccc}
C_{11} & C_{12} & \ldots & C_{1 i} & \ldots & C_{1 n} \\
\ldots & \ldots & \ldots & \ldots & \ldots & \ldots \\
C_{o 1} & \ldots & \ldots & C_{o i} & \ldots & C_{o n} \\
\ldots & \ldots & \ldots & \ldots & \ldots & \ldots \\
C_{l 1} & \ldots & \ldots & C_{l i} & \ldots & C_{l . n}
\end{array}\right]
$$

\section{Further DeVElopment to Mine VentiLAtion NETWORKS}

It is essential to include a source of energy for circulation of the air through the mine network. For that reason, a fan is normally installed at the entrance of the mine, normally a shaft or a tunnel. Sometimes, booster fans are also used on long airways deadlocks. The main fan is usually radial type while the booster fans are auxiliary type and installed inside the branches [13]. Furthermore, it is literally possible to add a new virtual loop to the network. The virtual loop (the dash line in Figure 1) connects the entrance airways to the exit airways and includes the main shafts and tunnels and a few branches. The airflow and pressure distributions in a ventilation network are controlled by suitably placing the fans, doors, and regulators [14]. Taking the virtual loop, as depicted in Figure 1, into consideration one would be able to further develop the energy conservation equations to [10]:

$$
\sum_{i=1}^{n} C_{o i} R_{i} Q_{i}^{2}-\sum_{i=1}^{n} C_{o i} P_{F i} \pm P_{n}=0(o=1,2, \ldots, l)
$$

Where: $P_{n}$ is the pressure due to natural ventilation. It takes a negative sign if the natural ventilation flow is in the same direction as the general flow, backing the air flowing clockwise in the loop; it otherwise takes a positive sign. The value of $P_{n}$ remains constant and is independent of airflow changes. Also $P_{F i}$ is the pressure due to the fan in branch $i$, whose value can be obtained using the fan characteristic function. Usually, the characteristic curve is given by the manufacturer and can be represented by equation (7) [10]:

$$
P_{F i}=a Q_{i}+b-c Q_{i}^{2}
$$




\section{THE NEWTON-RAPHSON METHOD}

In this method, a correction to the airflow $\left(\Delta_{o}\right)$ is defined and applied to all branches. The energy equation (4), therefore could be rewritten as equation (8) for the loop $o$, bearing in mind that $i$ is the number of branches in this loop. This equation is satisfied assuming that the correction factor $\left(\Delta_{o}\right)$ is applied to the existing airflows associated with all branches in the same loop. For a small value of $\left(\Delta_{o}\right)$ compared to $Q_{i}$ the additional terms vanish, and solving for $\left(\Delta_{o}\right)$ equation (10) will be obtained.

$$
\begin{gathered}
\sum_{i=1}^{n} C_{o i} R_{i}\left(Q_{i}+C_{o i} \Delta_{o}\right)^{2}=0(o=1,2, \ldots, l) \\
\sum_{i=1}^{n}\left(2 R_{i} Q_{i}+C_{o i} R_{i} Q_{i}{ }^{2}\right)=0 \\
\Delta_{o}=-\frac{\sum_{i=1}^{n} C_{o i} R_{i} Q_{i}{ }^{2}}{2 \sum_{i=1}^{n} R_{i} Q_{i}}
\end{gathered}
$$

The value for $\left(\Delta_{o}\right)$ is generalized in equation 11 :

$$
\Delta_{o}^{(m)}=-\frac{\sum_{i=1}^{n} C_{o i}^{(m-1)} R_{i} Q_{i}^{(m-1)^{2}}}{2 \sum_{i=1}^{n} R_{i} Q_{i}^{(m-1)}}
$$

Equation 11 should be satisfied for every loop in the network. In this equation, $i$ and $o$ denote branch and loop indices, respectively, and $m$ is the iteration number. The energy equation associated with the oth loop can be defined according to equation (12). The denominator in equation (11) is the derivative of numerator. Also, the numerator in equation (10) is the same as $F_{o}$ (equation 12). Therefore, a combination of the three equations $(10,11$, and 12) can be expressed in the matrix form (13):

$$
F_{o}=\sum_{i=1}^{n} C_{o i} R_{i} Q_{i}^{2}
$$

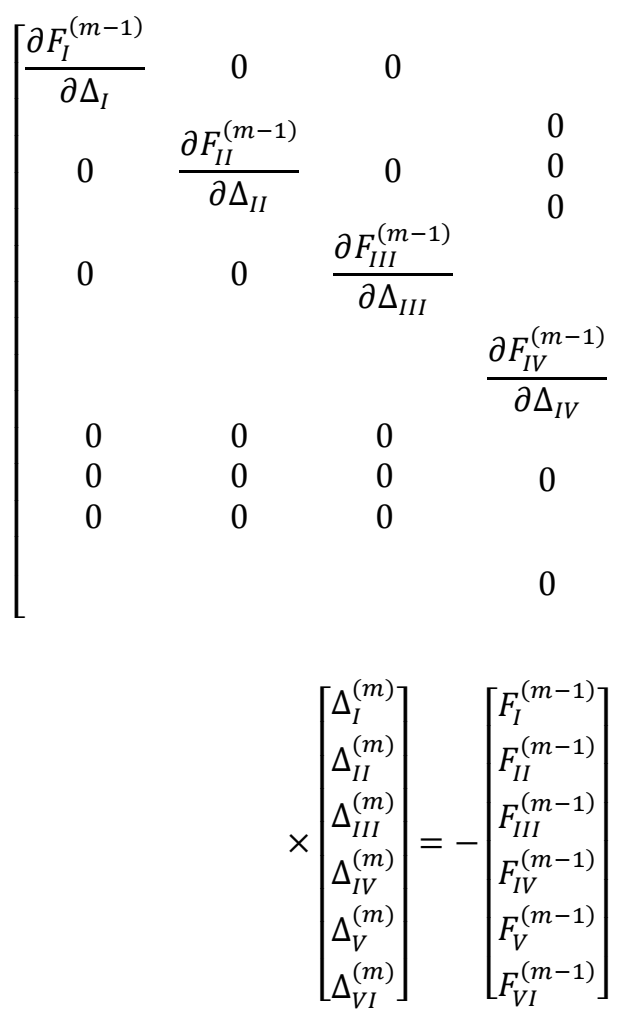

It is apparent that the approximation done for the individual loops is independent to those computed for the other loops. This approach, also known as Hardy-Cross method, is widely used due to simplicity. However, in the Newton - Raphson method, the value of $\left(\Delta_{o}\right)$ associated with the oth loop is determined taking those associated with the other loops into account at the same time. Similar computation has been done for correcting the air pressures associated with the nodes connecting all branches [15]. Also the corrections are done for the airflows associated with the common branches connecting the loops. The matrix form for this method is given in equation (14). As can be seen, the diagonal matrix (13) is now changed to a normal form (14) because the derivative of the energy equation is considered against all parameters involved in the iteration process. The approximation of the airflow in each loop in the $m$ th iteration is done according to the energy equation written for the same loop in the $(m-1)$ th iteration in addition to those of other loops.

$$
\left[\begin{array}{ccccccc}
\frac{\partial F_{I}^{(m-1)}}{\partial \Delta_{I}} & \frac{\partial F_{I}^{(m-1)}}{\partial \Delta_{I I}} & \frac{\partial F_{I}^{(m-1)}}{\partial \Delta_{I I}} & \frac{\partial F_{I}^{(m-1)}}{\partial \Delta_{I V}} & \frac{\partial F_{I}^{(m-1)}}{\partial \Delta_{V}} & \frac{\partial F_{I}^{(m-1)}}{\partial \Delta_{V I}} \\
\frac{\partial F_{I I}^{(m-1)}}{\partial \Delta_{I}} & \frac{\partial F_{I I}^{(m-1)}}{\partial \Delta_{I I}} & \frac{\partial F_{I I}^{(m-1)}}{\partial \Delta_{I I I}} & \frac{\partial F_{I I}^{(m-1)}}{\partial \Delta_{I V}} & \frac{\partial F_{I I}^{(m-1)}}{\partial \Delta_{V}} & \frac{\partial F_{I I}^{(m-1)}}{\partial \Delta_{V I}} \\
\frac{\partial F_{I I I}^{(m-1)}}{\partial \Delta_{I}} & \frac{\partial F_{I I}^{(m-1)}}{\partial \Delta_{I I}} & \frac{\partial F_{I I}^{(m-1)}}{\partial \Delta_{I I I}} & \frac{\partial F_{I I}^{(m-1)}}{\partial \Delta_{I V}} & \frac{\partial F_{I I I}^{(m-1)}}{\partial \Delta_{V}} & \frac{\partial F_{I I}^{(m-1)}}{\partial \Delta_{V I}} \\
\frac{\partial F_{I V}^{(m-1)}}{\partial \Delta_{I}} & \frac{\partial F_{I V}^{(m-1)}}{\partial \Delta_{I I}} & \frac{\partial F_{I V}^{(m-1)}}{\partial \Delta_{I I I}} & \frac{\partial F_{I V}^{(m-1)}}{\partial \Delta_{I V}} & \frac{\partial F_{I V}^{(m-1)}}{\partial \Delta_{V}} & \frac{\partial F_{I V}^{(m-1)}}{\partial \Delta_{V I}} \\
\frac{\partial F_{V}^{(m-1)}}{\partial \Delta_{I}} & \frac{\partial F_{V}^{(m-1)}}{\partial \Delta_{I I}} & \frac{\partial F_{V}^{(m-1)}}{\partial \Delta_{I I I}} & \frac{\partial F_{V}^{(m-1)}}{\partial \Delta_{I V}} & \frac{\partial F_{V}^{(m-1)}}{\partial \Delta_{V}} & \frac{\partial F_{V}^{(m-1)}}{\partial \Delta_{V I}} \\
\frac{\partial F_{V I}^{(m-1)}}{\partial \Delta_{I}} & \frac{\partial F_{V I}^{(m-1)}}{\partial \Delta_{I I}} & \frac{\partial F_{V I}^{(m-1)}}{\partial \Delta_{I I I}} & \frac{\partial F_{V I}^{(m-1)}}{\partial \Delta_{I V}} & \frac{\partial F_{V I}^{(m-1)}}{\partial \Delta_{V}} & \frac{\partial F_{V I}^{(m-1)}}{\partial \Delta_{V I}}
\end{array}\right]
$$

\section{THE LINEAR THEORY METHOD}

The Linear Theory is used for solving the algebraic and nonlinear differential equations. Equation (15) shows a common form of the Linear Theory [12]. In this equation, the relation of a function to the $(k+1)$ th power is given with the same function to the $k$ th power. This is the basis of Linear Theory method.

$$
\beta^{k} \psi^{k}=\beta_{0}^{k} \psi_{0}^{k}+\beta_{0}\left(\psi^{k+1}-\psi_{0}^{k}\right)+\psi_{0}\left(\beta^{k+1}-\beta_{0}^{k}\right)
$$

The Linear Theory function reconciles the airflows associated with all branches in successive iterations. The rate of convergence in this method is higher than those in the other two methods [17]. 
The initial values should not necessarily be given in this method. This is also an advantage, especially for large networks in which any errors in the initial guess may lead to substantial divergences. It is noteworthy to mention that the head losses in all branches, which were previously represented as nonlinear equations are now converted to linear equations, as in equation (16) [18].

$$
\Delta P_{i}=\left\lfloor R_{i} Q_{i 0}^{2-1}\right\rfloor Q_{i}=\left[R_{i} Q_{i 0}\right] Q_{i}=R_{i}^{*} Q_{i}
$$

The linear Theory method requires that some apparent changes to be done on the previously given energy equations (6). The term associated to the fans may become similar to that of the branch energy losses. For instance, the energy equation of the $o$-loop can be given as equation (17):

$$
\sum_{i=1}^{n} C_{o i} R_{i} Q_{i}^{2}-\sum_{i=1}^{n} C_{o i}\left(c G_{i}^{2}+P_{0}\right) \pm P_{n}=0(o=1,2, \ldots, l)
$$

$$
\begin{aligned}
& G_{i}=Q_{i}-\frac{a}{2 c} \\
& P_{0}=b+\frac{a^{2}}{4 c}
\end{aligned}
$$

Where $P_{n}$ is the natural ventilation pressure, which could be either in the same direction as the fan or in the opposite direction of the fan. Beside, for linearization of the term $c G_{i}^{2}$, equation (20) which is similar to equation (16) can be used [18].

$$
c G_{i}^{2}=c G_{i 0} G_{i}=K_{i} G_{i}
$$

Where $K_{i}$ is analogous to $R_{i}$ in equation (16). Then the number of linear equations will be the total number of loops and nodes. These equations will create a $n \times n$ system of linear equations as shown in matrix form (21):

$$
\left[\begin{array}{ccccc}
a_{11}^{(\mathrm{m}-1)} & a_{12}^{(\mathrm{m}-1)} & & \cdots & a_{116}^{(\mathrm{m}-1)} \\
a_{21}^{(\mathrm{m}-1)} & a_{22}^{(\mathrm{m}-1)} & & \cdots & a_{216}^{(\mathrm{m}-1)} \\
\vdots & \vdots & & & \vdots \\
a_{81}^{(\mathrm{m}-1)} & a_{82}^{(\mathrm{m}-1)} & \vdots & & a_{816}^{(\mathrm{m}-1)} \\
C_{11}^{(m-1)} R_{1}^{*(m-1)} & C_{12}^{(m-1)} R_{2}^{*(m-1)} & \cdots & C_{116}^{(m-1)} R_{16}^{*}(m-1) \\
C_{21}^{(m-1)} R_{1}^{*(m-1)} & C_{22}^{(m-1)} R_{2}^{*(m-1)} & \cdots & C_{216}^{(m-1)} R_{16}^{*}(m-1) \\
\vdots & \vdots & \vdots & \vdots \\
C_{61}^{(m-1)} R_{1}^{*(m-1)} & C_{62}^{(m-1)} R_{2}^{*(m-1)} & \cdots & C_{616}^{(m-1)} R_{16}^{*}(m-1)
\end{array}\right]_{16 \times 16}
$$

$$
\times\left[\begin{array}{c}
Q_{1}^{m} \\
Q_{2}^{m} \\
\vdots \\
Q_{16}^{m}
\end{array}\right]_{16 \times 1}=\left[\begin{array}{c}
0 \\
0 \\
\vdots \\
0 \\
C_{616}^{(m-1)}\left(P_{0}+R_{16}^{*}{ }^{(m-1)} \frac{a}{2 c}\right) \pm P_{n}
\end{array}\right]_{16 \times 1}
$$

In this equation system, the Node Continuity Equations can be obtained from multiplying the upper rows of the coefficient matrix by the variable matrix (i.e. the unknown values of the airflows). Also, the energy equations associated with each loop can be obtained from multiplying the lower rows of the coefficient matrix by the variable matrix. Note that the lowest row in the coefficient matrix, which includes the fans and the natural ventilation, is the most inclusive term exclusively introduced to the network. Some features of the matrix are as follows:

- It is defined to include most of the decisive terms in the network, where all the nodes and loops equations can be identified.

- More than one fan can be selected in the virtual loop, if needed. The natural ventilation which exists in many mines is included, and similar equations could be defined for those loops with fans or booster fans.

- The negative/positive effect of natural ventilation in accordance to that of the mechanical ventilation is also included.

The calculation will continue until the equation (22) is satisfied. Then, the operation stops and the final values associated with the branches' airflows will be the answers [19].

$$
\varepsilon=\frac{\sum\left|Q_{i}-Q_{i-1}\right|}{\sum Q_{i-1}} \leq 0.005
$$

\section{A NUMERICAL EXAMPLE}

These are for the ventilation network shown in Figure (1), which will be used in conjunction with equations (12), (13), and (22) to do a comparative study of the Newton-Raphson and Linear Theory methods. Also given is the main fan characteristic curve as in equation (23):

$$
H=-1084.51+29.28 Q-0.16 Q^{2}
$$

Table (1) shows the results obtained for all branches iteratively using the Newton-Raphson method. The values given in the first row (i.e. the $1^{\text {st }}$ iteration) are the initial guess quantities chosen according to the nodes continuity equations. Also shown in Table (2) are the error values from the NewtonRaphson method using equation (24):

$$
\delta=\frac{\sum\left|Q_{i}-Q_{i-1}\right|}{n} \leq 0.01
$$

To solve the example by the Linear Theory an identical initial value (i.e. the airflow $=100 \mathrm{~m}^{3} / \mathrm{s}$ ) for all branches is assumed. Table (3) shows the solved values obtained for all branches using the Linear Theory method. Also shown in Table (4) are the iterative error quantities resulted from the Linear Theory method using equation (24). 
TABLE I. The VALUES OBtAined FOR ALL BRANCHES Using THE NEWTON-RAPHSON METHOD

\begin{tabular}{|c|c|c|c|c|c|c|c|c|c|c|c|c|c|c|c|c|c|}
\hline \multicolumn{2}{|c|}{ Branch No. } & 1 & 2 & 3 & 4 & 5 & 6 & 7 & 8 & 9 & 10 & 11 & 12 & 13 & 14 & 15 & 16 \\
\hline \multicolumn{2}{|l|}{ Resistance } & 0.5 & 13.5 & 13 & 16.2 & 0.6 & 16.2 & 15.6 & 16.2 & 0.6 & 16.2 & 16.2 & 16.2 & 15.6 & 16.2 & 0.6 & 0.6 \\
\hline \multirow{6}{*}{ Iteration } & 1 & 100 & 50 & 25 & 47 & 50 & 25 & 25 & 22 & 53 & 50 & 62 & 40 & 25 & 13 & 12 & 100 \\
\hline & 2 & 145.67 & 46.25 & 41.52 & 44.80 & 99.42 & 38.16 & 4.73 & 3.28 & 100.87 & 42.89 & 38.73 & 35.45 & 61.26 & 65.42 & -4.16 & 145.67 \\
\hline & 3 & 129.44 & 43.52 & 47.16 & 40.80 & 85.92 & 35.31 & -3.64 & -6.36 & 88.64 & 31.67 & 30.48 & 36.84 & 50.61 & 51.80 & -1.19 & 129.44 \\
\hline & 4 & 123.59 & 42.07 & 46.54 & 40.21 & 81.52 & 34.64 & -4.47 & -6.33 & 83.38 & 30.17 & 29.88 & 36.21 & 46.88 & 47.17 & -0.29 & 123.59 \\
\hline & 5 & 123.10 & 41.94 & 46.43 & 40.12 & 81.16 & 34.54 & -4.49 & -6.61 & 82.98 & 30.06 & 29.82 & 36.13 & 46.61 & 46.85 & -0.23 & 123.10 \\
\hline & 6 & 123.10 & 41.94 & 46.43 & 40.12 & 81.16 & 34.54 & -4.49 & -6.31 & 82.98 & 30.06 & 29.82 & 36.13 & 46.61 & 46.85 & -0.23 & 123.10 \\
\hline
\end{tabular}

TABLE II. The ITERATIONS ERRORS OBTAINED By NEWTON-RAPHSON METHOD

\begin{tabular}{|l|l|l|l|l|l|l|}
\hline Iteration number & $\mathbf{1}$ & $\mathbf{2}$ & $\mathbf{3}$ & $\mathbf{4}$ & $\mathbf{5}$ & $\mathbf{6}$ \\
\hline Sum of the branches errors & - & 403.02 & 139.52 & 37.54 & 4.38 & $\leq 0.01$ \\
\hline Average of the branches errors & - & 25.19 & 8.72 & 2.35 & 0.27 & $\leq 0.01$ \\
\hline
\end{tabular}

TABLE III. The Solved VAlues ObTained For All Branches Using the Linear TheORy Method $\left(\mathrm{M}^{3} / \mathrm{S}\right)$

\begin{tabular}{|c|c|c|c|c|c|c|c|c|c|c|c|c|c|c|c|c|c|}
\hline \multirow{2}{*}{\multicolumn{2}{|c|}{$\begin{array}{l}\text { Branch No. } \\
\text { Resistance }\end{array}$}} & $\mathbf{1}$ & 2 & 3 & 4 & 5 & 6 & 7 & 8 & 9 & 10 & 11 & 12 & 13 & 14 & 15 & 16 \\
\hline & & 0.5 & 13.5 & 13 & 16.2 & 0.6 & 16.2 & 15.6 & 16.2 & 0.6 & 16.2 & 16.2 & 16.2 & 15.6 & 16.2 & 0.6 & 0.6 \\
\hline \multirow{10}{*}{ Iteration } & 1 & 100 & 100 & 100 & 100 & 100 & 100 & 100 & 100 & 100 & 100 & 100 & 100 & 100 & 100 & 100 & 100 \\
\hline & 2 & 151.09 & 47.35 & 43.07 & 44.05 & 103.74 & 35.76 & 4.28 & 0.99 & 107.03 & 40.04 & 35.53 & 34.54 & 67.96 & 72.49 & $\overline{4} .51$ & 151.09 \\
\hline & 3 & 163.47 & 56.63 & 52.09 & 54.17 & 117.38 & 44.93 & 8.26 & 4.83 & 121.52 & 43.39 & 41.73 & 39.25 & 79.17 & 83.32 & $\begin{array}{l}- \\
7.41\end{array}$ & 163.47 \\
\hline & 4 & 146.25 & 45.64 & 50.36 & 43.28 & 108.74 & 39.80 & $\overline{2} .34$ & 1.35 & 95.45 & 37.46 & 33.29 & 34.64 & 61.65 & 61.81 & 6.17 & 146.25 \\
\hline & 5 & 131.14 & 42.68 & 48.17 & 41.42 & 86.76 & 36.12 & $\overline{5} .49$ & 7.75 & 89.02 & 30.63 & 29.57 & 37.32 & 50.64 & 51.70 & 1.06 & 131.14 \\
\hline & 6 & 127.38 & 42.35 & 47.65 & 41.62 & 85.33 & 35.48 & - & -6.53 & 88.56 & 31.18 & 30.07 & 36.60 & 51.85 & 52.96 & $\overline{1.11}$ & 127.38 \\
\hline & 7 & 124.58 & 42.43 & 45.53 & 40.68 & 82.53 & 34.93 & - & $\begin{array}{l}- \\
6.24 \\
\end{array}$ & 84.38 & 31.02 & 29.76 & 36.29 & 47.52 & 48.76 & 0.59 & 124.58 \\
\hline & 8 & 123.59 & 42.05 & 46.54 & 40.21 & 81.53 & 34.64 & - & -6.33 & 83.37 & 30.15 & 29.87 & 36.20 & 46.90 & 47.17 & 0.28 & 123.59 \\
\hline & 9 & 123.10 & 41.94 & 46.43 & 40.12 & 81.16 & 34.54 & $\begin{array}{l}- \\
4.49 \\
\end{array}$ & - & 82.98 & 30.06 & 29.82 & 36.13 & 46.62 & 46.85 & $-\overline{0.23}$ & 123.10 \\
\hline & 10 & 123.10 & 41.94 & 46.43 & 40.12 & 81.16 & 34.54 & $-\overline{4}$ & $-\overline{6.31}$ & 82.98 & 30.06 & 29.82 & 36.13 & 46.62 & 46.85 & $\overline{0} .23$ & 123.10 \\
\hline
\end{tabular}

TABLE IV. THE ITERATIVE ERROR QUANTITIES RESUlTED FROM THE LINEAR THEORY METHOD

\begin{tabular}{|l|l|l|l|l|l|l|l|l|l|l|}
\hline Iteration number & $\mathbf{1}$ & $\mathbf{2}$ & $\mathbf{3}$ & $\mathbf{4}$ & $\mathbf{5}$ & $\mathbf{6}$ & $\mathbf{7}$ & $\mathbf{8}$ & $\mathbf{9}$ & $\mathbf{1 0}$ \\
\hline Sum of the branches errors & - & 891.40 & 137.50 & 171.22 & 121.03 & 17.80 & 26.80 & 10.05 & 3.04 & $\leq 0.01$ \\
\hline Average of the branches errors & - & 55.71 & 8.59 & 10.70 & 7.56 & 1.11 & 1.67 & 0.63 & 0.19 & $\leq 0.01$ \\
\hline
\end{tabular}

As can be seen, the Newton-Raphson method would yield a satisfactory error after five iterations. However, the Linear Theory method would do the same after nine iterations. This implies a higher rate of convergence for the Newton's method.

The initial guess for $Q_{i 0}$ is considered to be equal to the average of the apparent final answers. Because, when this guess, which is the same for all the branches, differs significantly from the average of the final answer it will be less likely to suppress divergence in the iteration process. Such divergence is likely to occur when fans are used in the network $^{4}$. It has also been shown that to reduce the likelihood of oscillation for the real answers the mean of the two previous iterations may be used for every branch in the sequence [18].

For comparison, the iteration error against the number of iterations is plotted in Figure 2 for both methods. 


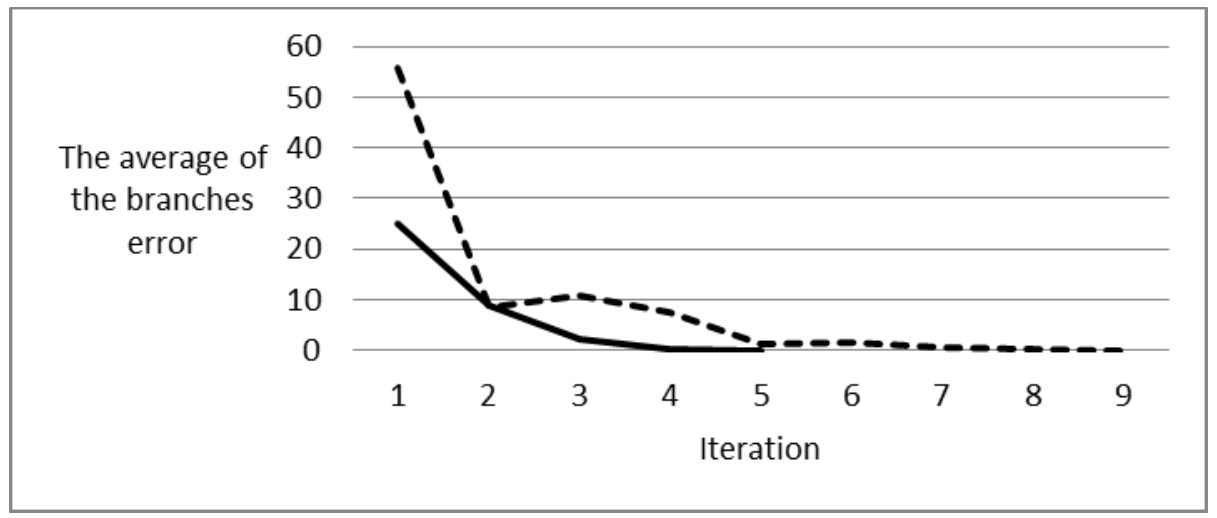

Fig. 2. The iteration error against the number of iterations for the Newton-Raphson method (continuous line) and the linear Theory method (discrete line)

As can be seen in Figure 2, in the first iteration implemented by the Newton-Raphson method, the value of the error is lower in magnitude. It also decreases smoothly to yield the answer. In the linear Theory method, the iteration error starts with higher value and changes with some oscillation to reach the answer. This oscillation can lead to divergence in large networks. It is suggested that in the Linear Theory the next trial solution should be the average of the previous two solutions equation (25):

$$
Q_{n}=\frac{Q_{n-1}+Q_{n-2}}{2}
$$

Alternatively, it may be suggested equation (26):

$$
Q_{n}=\sqrt{Q_{n-1} \times Q_{n-2}}
$$

which works just as satisfactorily in removing the oscillatory nature of successive solutions.

It can adequately deal with large systems of equations and has the advantage that the first approximation to the solution does not need to satisfy continuity conditions and the initial values may be arbitrary. Wood and Charles [20] observed an oscillatory nature in successive estimates close to the converged solution and suggested the next trial solution to be the average of the previous two solutions.

\section{CONCLUSION}

The large number of variables in mine ventilation networks implies the need for using the approximate methods. The matrices of nodes and loops are shown to be good characteristics of the network. Using the equations in the matrices form and applying the approximate methods based on iteration could be the core of simple programming tasks in mine ventilation network analysis. The possibility of the initial guesses for the branch airflow being incorrect in the NewtonRaphson method is lower. This is because, in comparison with the water and gas networks, the mine ventilation networks are less expanded. Therefore, in comparison with the Linear Theory method, the Newton-Raphson method converges faster. On the other hand, when using computational tools and software the advantage of faster convergence becomes less important and therefore the Linear Theory method will be more preferred.

\section{ACKNOWLEDGMENT}

The authors would like to thank Imam Khomeini International University for the support provided to carry out this research.

\section{REFERENCES}

[1] Cross H. Analysis of Flow in Networks of Conduits or Conductors. Engineering Experiment Station, University of Illinois, Urbane, 1936; 286- 295.

[2] Scott DR, Hinsley FB. Ventilation Network Theory. Colliery Engineering, London, 1951.

[3] Wood DJ, Charles COA. Hydraulic Network Analysis Using Linear Theory. Journal of Hydraulics Division, ASCE, 1972; 98(HY7): 11571170.

[4] Jeppson RW, Tavallaee A. Pumps and Reservoirs in Networks by Linear Theory. Journal of Hydraulics Division, ASCE, 1975; 101(HY7): 576580.

[5] Bhamidipati SS, Procarione JA. Linear Analysis for the Solution of Flow Distribution Problems in Mine Ventilation Network. Proceedings of the North American 9th US Mine Ventilation Symp, 1985, 646-654.

[6] M. Kolarczyk, "Determination of a matrix column of air volume output sensitivity to changes in side branches resistance in a mine ventilation network," GeoScience Engineering, Vol. LIII, No. 1, pp 25-35, 2007.

[7] X. Star Wu and E. Topuz, "Analysis of Mine Ventilation Systems Using Operations Research Methods," Int. Trans. Opl Res., Vol. 5, No. 4, pp. 245-254, 1998.

[8] S. Yuna and W. Hai-ning, "Study and Application on Simulation and Optimization System for the Mine Ventilation Network," Procedia Engineering, Vol. 26, pp 236 - 242, 2011.

[9] F. Wei, Z. Fangping, and L. Huiqing, "The use of 3D simulation system in mine ventilation management," Procedia Engineering, Vol. 26, pp. 1370-1379, 2011.

[10] McPherson MJ. Subsurface Ventilation and Environmental Engineering. Chapman \& Hall, UK, 1993.

[11] Yun QX, Huang KM. Determination of Ventilation System for Mines by Genetic Algorithm. Proceedings of the $6^{\text {th }}$ Environmental Issues and Management of Waste in Energy and Mineral Production Symp, , 2000, 725-733.

[12] Madani H, Maleki B. Analysis of Mines Ventilation Network by Newton-Raphson method based on the $\mathrm{H}$ equations. Journal of Mine Eng. 2008; 3(5): 71-77.

[13] Hartman HL, Mutmansky JM, Wang YJ. Mine Ventilation and Air Condition. Wiley, 1982.

[14] Lowndes IS, Fogarty T, Yang ZY. The application of genetic algorithms to optimize the performance of a mine ventilation network: the influence of coding method and population size. Springer-Verlag, 2004.

[15] Madani H, Maleki B. Analysis of Mines Ventilation Network by Newton-Raphson method based on the $\Delta Q$ equations. Journal of AmirKabir University, 2007; 66c. 
[16] Phillips GM, Taylor PJ. Theory and Applications of Numerical Analysis. Academic Press, 1980.

[17] Jeppson RW. Steady Flow Analysis of Pipe Networks, An Instructional Manual, Utah State University, 1974.

[18] Ellis DJ, Simpson AR. Convergence of Iterative Solvers for the Simulation of a Water Distribution Pipe Network. Department of Civil and Environmental Engineering, The University of Adelaide, Research
Report No. R138, 1996.

[19] Savic DA, Walters GA. An Evolution Program for Pressure Regulation in Water Distribution Networks. Report Number: 94/15, University of Exeter, United Kingdom, 1994.

[20] Wood DJ, Charles COA. Optimization of mine ventilation and methane recovery. European Commission technical coal research Mining operations, EUR 17392 EN, 1993. 